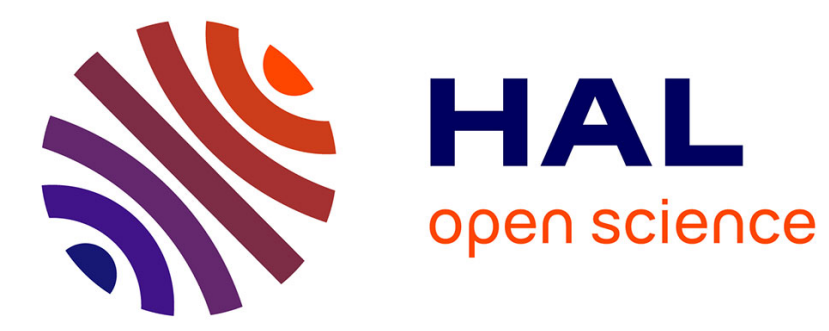

\title{
Perceptual investigation of prosodic phrasing in French
}

\author{
Laury Garnier, Lorraine Baqué, Anne Dagnac, Corine Astésano
}

\section{To cite this version:}

Laury Garnier, Lorraine Baqué, Anne Dagnac, Corine Astésano. Perceptual investigation of prosodic phrasing in French. Speech Prosody 2016, May 2016, Boston, United States. hal-01330866

\section{HAL Id: hal-01330866 https://hal.science/hal-01330866}

Submitted on 13 Jun 2016

HAL is a multi-disciplinary open access archive for the deposit and dissemination of scientific research documents, whether they are published or not. The documents may come from teaching and research institutions in France or abroad, or from public or private research centers.
L'archive ouverte pluridisciplinaire HAL, est destinée au dépôt et à la diffusion de documents scientifiques de niveau recherche, publiés ou non, émanant des établissements d'enseignement et de recherche français ou étrangers, des laboratoires publics ou privés. 


\title{
Perceptual investigation of prosodic phrasing in French
}

\author{
Laury Garnier $^{1,2}$, Lorraine Baqué ${ }^{3}$, Anne Dagnac ${ }^{2}$, Corine Astésano ${ }^{1}$ \\ ${ }^{1}$ URI Octogone- Lordat (.E.A 4156), Université de Toulouse, UT2J, France \\ ${ }^{2}$ CLLE (UMR 5263), Université de Toulouse, CNRS, UT2J, France \\ ${ }^{3}$ Laboratori fLexSem, Universitat Autònoma de Barcelona, Spain \\ laury.garnierduniv-tlse2.fr, lorraine.baque@uab.cat, dagnacduniv-tlse2.fr, astesano@univ- \\ trse2.fr
}

\begin{abstract}
The aim of this paper is to investigate prosodic phrasing and more precisely the use of prosodic cues in the marking of morphosyntactic units in French. As a first step towards this goal, a perception study was conducted on 27 listeners, who had to perform 3 distinct perceptual tasks on 32 syntactically controlled phrases read by a female speaker: a prominence strength judgment task, a boundary strength judgment task, and a task where listeners had to choose between 4 different phrase groupings intended to reflect the potential choices of prosodic phrasing. The corpus consists of syntactically ambiguous structures manipulating high and low adjective attachment on 2 coordinated nouns. It was designed to specifically test the role of prominence and boundary cues in the marking of prosodic constituency. Our results show that listeners use prosodic cues to discriminate between the two syntactic structures, with boundary cues being more readily used to capture morphosyntactic structuring. More interestingly, our results indicate that prominence and boundary cues are used to distinguish finer-grained grouping levels than those predicted by traditional descriptions on French prosodic structure.
\end{abstract}

Index Terms: prosodic phrasing, French, perception, prominence, boundary, grouping.

\section{Introduction}

One of the most important functions of prosody is to segment the speech flow into organized units. Prosodic cues such as prominences and boundaries are used to structure speech and partake in what is referred to as 'prosodic phrasing'. In doing so, prosodic cues help linearize constraints arising from other linguistic levels such as semantic-pragmatic constraints and syntactic rules [1;2]. A long line of research has been specifically interested in establishing the relationship between prosody and syntax. While most prosodists do not deny some interdependencies between prosody and syntax, it is arguably difficult to find common grounds on a precise relationship between the two linguistic components. Whether one assumes a mapping between syntax and prosody, whereby prosodic cues mark morphosyntactic units, or not, it is difficult to find a clear consensus on the levels reflecting prosodic hierarchy.

Indeed, there is a wide array of propositions ranging from 2 levels (Accentual Phrase or ap, and Intonational Phrase or $I P$ : [3]) to 5 levels (including according to models: Prosodic/Phonological Word or pw, Clitic Group, Phonological Phrase or PP, Intonational Phrase and Utterance; [4]-[7]). Some authors, however, propose to envisage the possibility of recursive prosodic units in order to better capture the link between prosody and syntax [8; 9]. In these proposals, Super-Major Phrase (corresponding to an $I P$ containing several $I P s$ ) can be found to posit an intermediate level between the Utterance and the Major Phrase. This recursion principle has been captured in more recent propositions revising the Strict Layer Hypothesis in the frame of the Optimality Theory [10; 11]. Also, a revision of the initial model by Selkirk [4] proposed to divide the $P P$ level into 2 distinct levels: a Minor PP and a Major PP [12], equivalent to the $a p$ and the Intermediate Phrase (ip) levels, respectively, as proposed in [13]. Discussions on the existence of an intermediate level in French have also arisen, with propositions that the ip exists for specific syntactic structures, such as tag questions and dislocated structures [14] (see also the 'segment d'unité intonative' in [15]). Further evidence points towards a true intermediate level applying to longer constituents and situated between the $a p$ and the $I P$ in French [16], more in line with the initial proposition in [13].

For French, this question about which levels are necessary to account for prosodic phrasing is of particular interest. While stress and boundary tones are clearly dissociated in English, (final) stress is syncretic to boundary tones in French, both aligning to the right edge of prosodic domains. Stress in French is also said to be post-lexical, marking the $a p$ rather than the $p w^{l}$. Both these characteristics have led some authors to question the phonological status of the final accent $(F A)$ and to characterize French as a 'language without accent' [18] or a 'boundary language' $[19 ; 20]$. Following up on this view, $F A$ is described as a pitch accent $\left(H^{*}\right)$ found to mark the lowest level of the hierarchy (ap), which however disappears in favor of the boundary tone $(H \%)$ at the higher $(I P)$ level of the hierarchy [3]. Thus, intonation pre-empts stress at higher levels of the prosodic hierarchy. Another type of accent has also been described to mark the $a p$ level in French: the initial accent $(I A)$, said to be secondary and optional as opposed to the primary $F A$. IA has been described as a mere rhythmic device occurring on longer constituents (see among others [19; $21 ; 22])$. Descriptions of its role in the marking of the prosodic hierarchy are scarce and its functions not well established. Its functions were specifically studied on a corpus controlling for constituents' lengths and syntactic structure [23]. This acoustic study revealed that the function of $I A$ is more one of structuration than rhythmic balancing. It was also shown to

${ }^{1} p w$ sometimes refers to the Clitic Group of $[5 ; 6]$, hence similar to the ap. Here, we refer to the sense of [5; 6] and [17], corresponding to the Lexical Word. 
more readily mark structure than $F A$ at lower levels of prosodic constituency, close to the $a p$ and possibly the $p w$. A perception study on a subset of the same corpus confirmed these results [24]. They showed that French listeners can perceive $I A$ and $F A$ independently from boundaries, with $I A$ being perceived as consistently stronger than $F A$ throughout the prosodic hierarchy.

The present study is a follow up of [24]. It is not intended to address syntax/prosody mapping per se; rather, it is specifically designed to tackle the issue of which levels of prosodic structure are necessary to account for prosodic phrasing in French. Perception is used here as an interface between the acoustic signal and phonology, in line with propositions in [25]-[27]. It is also used to circumvent the variability or 'flexibility' of prosodic cues found in the signal to instantiate constituency at predicted similar levels, as described in $[1 ; 2 ; 28]$.

\section{Method}

\subsection{Corpus}

The linguistic material used for this perception study derives from the Edinburgh Corpus [23]. This corpus is composed of syntactically ambiguous structures that can be disambiguated via prosodic cues (prominences, boundary tones, pauses). These structures are composed of two coordinated nouns (NI $\& N 2$ ) and an adjective (A). Syntactic ambiguity is created by manipulating the adjective scope, with low or high syntactic attachment of the adjective, yielding 2 syntactic conditions and the following phrasing (with predicted structure's depths):

Condition1 - The adjective qualifies only the second noun $[(N I)]_{i p}\left[\left(N 2_{p w} A\right)\right]$, predicting a low syntactic attachment of the $A$ with an Intermediate Phrase (ip) boundary between N1 and N2 and a Prosodic Word ( $p w$ ) boundary between N2 and A.

Condition 2 - The adjective qualifies both nouns $\left[(N I)_{q}(N 2)\right]_{p}$ $[(A)]$, predicting a high syntactic attachment of $A$ with an Accentual Phrase (ap) boundary between $N 1$ and $N 2$ plus an Intermediate Phrase (ip) boundary between $N 2$ and $A$.

The prosodic structure proposed here is composed of 3 levels under the IP: $i p$, $a p$ and $p w$. While $a p$ is largely accepted in French, $i p$ is more controversial and $p w$ is hardly ever mentioned. However, in earlier acoustic/perception studies $[23 ; 24]$ the question of the relevance of the $p w$ unit was raised. The present study aims at further investigating this finer granularity in the prosodic hierarchy. The corpus is also manipulated with regards to constituents' length; nouns and adjectives increase from 1 to 4 syllables, in all possible combinations. $\mathrm{N} 1$ and $\mathrm{N} 2$ lengths always co-vary. The original corpus is composed of 4 sets of phrases. Each phrase is extracted from a carrier sentence, the syntactic structure of which enforces a major prosodic boundary after $A$, realized as a non-terminal, high $I P$ boundary. Morphology and semantics are controlled so that the adjective can apply to both nouns (see [23] for more details on the corpus).

In this perception study, one set of phrases read by one female speaker was used: 32 phrases with 4 lengths of $N$ and 4 lengths of $A$ in the 2 different syntactic conditions. Our data analysis however excludes phrases with monosyllabic words, because they do not allow for the distinction between initial accents $(I A)$ and final accents $(F A)$. Our results were thus computed on 24 phrases, namely 12 phrases by condition.

\subsection{Participants and experimental tasks}

Each sequence $N 1+N 2+A$ was perceptually judged by 27 French native listeners. They performed 3 perception tasks:

Boundary task: listeners had to judge the degree of break between each word (5 potential sites) on a scale from 0 (no break) to 3 (strong break).

Prominence task: listeners had to judge the degree of salience of each syllable of the phrases (ranging from 6 to 15 potential sites depending of the combination of constituents' length), on a scale from 0 (no salience) to 3 (strong salience).

Grouping task: listeners had to judge how the sequences $N 1+N 2+A$ are divided into groups by selecting between 4 different phrase groupings $(G 1, G 2, G 3, G 4)$ intended to reflect the possible choices of prosodic phrasing (see Figure1).
G1 les lumières et les balises vertigineuses
G3 les lumières et les balises vertigineuses
G2 les lumières et les balises vertigineuses
G4 les lumières et les balises vertigineuses

Figure 1: The 4 groups proposed in the Grouping task.

The order of presentation of the 3 tasks was counterbalanced between listeners. For each task, phrases were presented via headphones on a computer and their presentation was randomized across listeners. Participants could listen to the same phrase up to 5 times in order to perform their scoring, by pressing a 'play' button on the computer. A training period was undertaken before the start of the experiment, using 4 phrases from another set of $N 1+N 2+A$ sequences than the one used for the present perception study.

\subsection{Predictions}

As exposed in the introduction, our perception study is designed to clarify the role of prosodic cues in the marking of morphosyntactic units.

In the first two perception tasks, boundaries and prominences are scored separately to capture the relative effect of these two distinct phonological events on phrasing. In the third task, groups are used to more specifically investigate prosodic phrasing strategies and to help capture the underlying factors explaining potential mismatches with syntax. G1 and $G 2$ correspond to the predictions of the syntactic Conditions 1 and 2. By contrast, $G 3$ and $G 4$ were chosen to propose alternative groupings for Conditions 1 and 2 , so as to help uncover potential finer-grained boundary levels. G3 can be found in both syntactic predictions, but would reflect different relative boundary strengths in Condition $1(N 1 \| N 2 \mid A)$ and 2 $(N 1 \mid N 2 \| A)$. G4 may also correspond to both conditions but cannot reflect gradual boundary levels within the IP. G3 and $G 4$ were also intended to get perceptual insight on previous acoustic results [23] on the same corpus, showing preferential marking of structure by $I A$ over $F A$. G3 will be used to perceptually test these findings, where $I A$ was found to largely mark $N 2(83 \%)$ in Condition2 (after a predicted ap boundary), and marginally mark $A(23 \%)$ in Condition 1 (after a predicted $p w$ boundary). $G 4$ is intended to test the potential perception of no boundary between constituents, despite the common IA marking of $N 2$ and $A$.

Ultimately, the choice of one grouping over another will be correlated to the results emerging from the boundary and prominence tasks, with the goal to clarify the respective or conjoined implication of these prosodic phenomena in speech structuration. 


\subsection{Data selection and analysis}

The design of our data supposes that we first investigate listeners' interpretation of Conditions 1 and 2 in terms of the 4 grouping choices.

\subsubsection{Syntactic condition effect on Groups}

The relationship between Conditions and Groups was investigated using a chi-square test of independence. Results show a significant effect of Condition on Group $\left(\chi^{2}(3,648)=530.89, p<.001\right)$. Post-hoc analyses show that $G 1$ is highly associated to Condition 1 (93.5\% vs. G2: $0.6 \%, G 3$ : $5.9 \%$ and $G 4: 0.0 \%$; adjusted $p$-value<.001), while Condition2 is significantly more frequently associated to $G 4(42.9 \%), G 2$ (32.1\%) and $G 3(21.0 \%)$ than to $G 1$ (4.0\%; adjusted $p$ value <.001). For lack of data, we thus decided to exclude from further analyses the cases in which Condition 1 was perceived as G2, G3 or G4, and those in which Condition2 was perceived as $G 1$. Listeners are able to perceptively distinguish the two syntactic conditions: $G 1$ massively corresponds to Condition1, while $G 2$ is associated to Condition2. Finally, whereas we expected $G 3$ and $G 4$ to be distributed equally over the two conditions, they are mostly associated to Condition 2 .

\subsubsection{Statistical models}

On the basis of these results, we analyzed the effect of Group (G1, G2, G3 and G4) on Prominence and Boundary scores (ranging from 0 to 3 ). Constituents' lengths were taken into account for $N$ only, while $A$ lengths were collided in order to increase statistical power. Analyses focused on the sites in the phrases reflecting structuring, i.e. between $N 1 \mid N 2$ and between $N 2 \mid$ A. Boundary scores were measured after each content word, and Prominence scores were measured immediately before boundaries (FA on N1 and N2) and at the beginning of content words after boundaries (IA on N2 and A). Separate Mixed Linear Models were computed for dependent variables Boundary and Prominence respectively, and with subjects and phrases as random variables. The predictors were Groups and $N$ length (2, 3 and 4 syllables). The non-significant variables or interactions were excluded from the final models.

\section{Results}

\subsection{Boundaries}

\subsubsection{N1|N2 boundary scores}

There was a main effect of Group $(\mathrm{F}(3,91.25)=494.14$, $p<.001)$ and of $N$ length $(\mathrm{F}(2,37.54)=10.59, p<.001)$, with an interaction between the two predictors $(\mathrm{F}(6,89.32)=4.04$, $p<.001)$. Post-hoc analyses show significant differences ( $p<.05)$ of boundary scores between groups, for all $N$ lengths' conditions: the boundary in $G 1$ (mean score $(\mathrm{ms})$ range: 3.383.62 ) is perceived as much stronger than in the other groups ( $m s$ ranges: $G 2=1.44-1.83 ; G 3=1.43-2.21 ; G 4=1.36-1.60$ ). In addition, the boundary in $G 3$ for 2 and 4 syllables $N$ is also perceived as stronger ( $m s: 2.10$ and 2.21 respectively) than in $G 2$ ( $m s: 1.58$ and 1.83 respectively) and $G 4$ ( $m s: 1.40$ and 1.60 respectively). For 3 syllables $N$, however, no difference is observed between the boundaries in $G 3$ ( $m s: 1.43$ ), G2 ( $m s$ : $1.44)$ and $G 4$ (ms: 1.36). Altogether, these results indicate that listeners perceive 3 different boundary levels between $N 1$ and $N 2$ : the strongest in $G 1$, an intermediate one in $G 3$ and the weakest one in $G 2$ and $G 4$.

\subsection{2. $\quad$ 2| A boundary scores}

Our analysis shows a main effect of Group ( $\mathrm{F}(3$, $111.64)=30.95, p<.001)$. Post-hoc analyses show significant differences $(p<.05)$ for all comparisons except between $G 2$ and G3. The boundaries in $G 3$ (ms: 2.52) and $G 2$ (ms: 2.42) are perceived as much stronger than in $G 4$ (ms: 1.99), with the lowest boundary score for $G 1$ ( $m s: 1.77)$. Again, it seems that listeners perceived 3 different boundary levels between $N 2$ and $A$ : the strongest in $G 2$ and $G 3$, an intermediate one in $G 4$ and the weakest one in $G 1$.

\subsubsection{Relative strength of $N 1 \mid N 2$ and $N 2 \mid$ A boundaries}

Prosodic phrasing is better accounted for when capturing the syntagmatic relationship between boundaries. This relationship was inferred in our data by subtracting Boundary scores perceived between $N 2 \mid A$ to those perceived between $N 1 \mid$ N2. Results show main effects of Group $(\mathrm{F}(3,106.96)=278.10$, $p<.001)$ but no effect of $N$ length. Post-hoc analyses show that the only significant contrasts $(p<.001)$ are between $G 1$ ( $m s:-1.78)$ and the other three groups ( $m s: G 2=0.96$; $G 3=$ 0.47 and $G 4=0.50)$. For $G 1$, the second boundary $(N 2 \mid A)$ is perceived as much weaker than the first boundary $(N 1 \mid N 2)$. By contrast, for $G 2, G 3$ and $G 4$, the second boundary $(N 2 \mid A)$ is perceived as stronger than the first boundary $(N 1 \mid N 2)$. It seems that listeners perceive two types of syntactic/prosodic grouping with a different balance of boundary strengths: strong + weak boundaries $(N 1|N 2>N 2| A)$ in Condition1 associated to $G 1$, and weak + strong boundaries $(N 1|N 2<N 2| A)$ in Condition2 associated to $G 2, G 3$ and $G 4$.

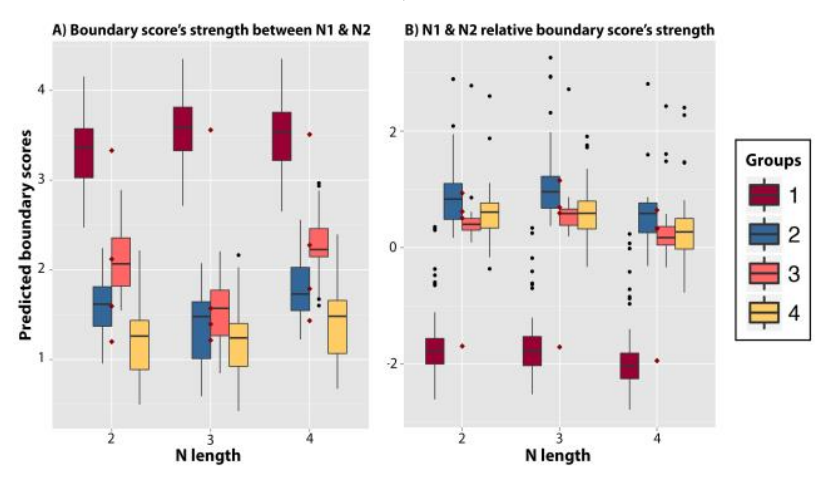

Figure 2: Perception results as a function of $N$ length and Groups for A) N1|N2 boundary scores' strength, and $B)$ Relative strength of $N 1 \mid N 2$ and $N 2 \mid A$ boundaries.

\subsection{Prominences}

\subsubsection{Final and Initial Accents}

For $F A$ on $N 1$, our analysis shows main effects of Group $(\mathrm{F}(3,568.71)=6.24, p<.001)$ and of $N$ length $(\mathrm{F}(2,556.49)=8.14$, $p<.001$ ). Post-hoc analyses show that $F A$ on $N 1$ in $G 1$ ( $m s$ : $2.39)$ is perceived as stronger $(p<.035)$ than in $G 2$ (ms: 2.09) and $G 4$ ( $m s: 2.17$ ), while the difference with $G 3$ (ms: 2.13) doesn't reach significance $(p=.087)$. The other contrasts are not significant. As far as $N$ length effect is concerned, $F A$ on $N 1$ is globally perceived as significantly weaker $(p<.014)$ when $N$ is composed of 3 syllables (ms: 2.03) as compared to nouns of 2 or 4 syllables ( $m s: 2.25$ and 2.31 respectively). No difference is observed between $N$ of 2 and 4 syllables. For $F A$ on $N 2$, our analysis shows no effect of Group and a main 
effect of $N$ length $(\mathrm{F}(2,42.64)=3.87, p=.029)$. Post-hoc analyses show that $F A$ on $N 2$ is perceived as weaker when $N$ is composed of 3 rather than 4 syllables ( $m s: 1.92$ vs 2.14 respectively, $p<.022)$. For $I A$ on $N 2$, there are no significant main effects of Group and $N$ length ( $p>.05$; $m s$ range: 2.262.80). The same goes for $I A$ on $A$ ( $m s$ range: 2.00-2.72).

\subsubsection{IA and FA relative prominence strengths}

The question remains as to the relative weight of prominences in the marking of the structure. We computed a paired twotailed t-test with $I A$ and $F A$ scores around the two boundary sites, for each group and independently from $N$ length. Results show a significant difference between $F A$ on $N 1$ and $I A$ on $N 2$ : $I A$ is perceived as stronger than $F A$ in $G 3$ ( $m s: 1.63$ vs. 1.07; $t(67)=3.30, p=.002)$ and in $G 4(m s: 1.56 v s .1 .07, t(138)=5.55$, $p<.001) . I A$ is also marginally stronger than $F A$ in $G 2$ ( $m s$ : 1.34 vs. $1.11 ; t(103)=1.96, p=.053)$. There is however no effect for $G 1(p=.338)$. Our results also show a significant difference between $I A$ on $A$ and $F A$ on $N 2$ for all groups: $I A$ is perceived as stronger than $F A$ in $G 1$ ( $m s: 1.23$ vs. $0.92 ; t(302)=4.83$, $p<.001)$, in $G 2$ ( $m s: 1.38$ vs. $0.99 ; t(103)=3.30, p=.001)$, in $G 3$ $(m s: 1.45$ vs. $0.82 ; t(67)=3.80, p<.001)$ and in $G 4(m s: 1.42 v s$. $1.04 ; t(138)=4.06, p<.001)$.

\section{Discussion \& Conclusion}

The aim of this study was to investigate prosodic phrasing in French, and more precisely the use of prosodic cues in the marking of morphosyntactic units. Indeed, as exposed in the introduction, there is no clear consensus on how many levels are necessary to reflect prosodic hierarchy, especially for French descriptions. Investigating prosodic phrasing is best done manipulating syntactic ambiguity, as shown in the seminal work by Lehiste [29; see also 28]. Our corpus uses low and high adjective attachment to two conjoined nouns in order to elicit prosodic disambiguation in French. It also manipulates constituents' length. If structure depth can be uncovered by means of acoustical analyses, some authors have however demonstrated the difficulty of matching acoustic features to syntactic structures [28]. Perceptual investigations of such a link might thus be a better gateway towards this end [25]-[27], a useful interface between acoustic cues and phonology. Our participants were asked to perform three separate perceptual judgments: two tasks (Boundary and Prominence strengths' scoring) were designed to investigate the role of these prosodic cues in structure marking. Our statistical design aimed at interpreting these results with regard to the Grouping task, in order to more specifically test their relationship to prosodic phrasing.

Taken together, our results indicate that listeners are able to distinguish the two intended syntactic conditions: while Condition 1 was exclusively associated with $G 1$, Condition2, however, was distributed across G2, G3 and G4. Prosodic phrasing is thus perceived with different degrees of granularity in Condition2. This granularity could be investigated by testing the effect of each Grouping choices on Boundary and Prominence scores independently. Altogether, our results indicate that morphosyntactic structuring is better accounted for by Boundary than Prominence perception. Indeed, while Grouping choices very marginally affect Prominence perception, they largely explain Boundary scores. Indeed, Grouping choices indicate that listeners perceive various Boundary levels on each site $(N 1|N 2 ; N 2| A)$. However, a syntagmatic investigation indicates that listeners adjust relative boundary strengths in order to maintain the intended syntactic structure: strong + weak boundary $(N 1|N 2>N 2| A)$ in Condition 1 and weak + strong boundary $(N 1|N 2<N 2| A)$ in Condition2. Confronting the syntagmatic analysis with the analysis by site allows for finer interpretation of the granularity of these two boundary strengths: listeners perceive up to three boundary levels on each site below the $I P$ level. Between $N 1$ and $N 2$, the strongest boundary perceived in $G 1$ $(N 1|N 2>N 2| A)$ could reflect an ip boundary. The weaker one perceived in $G 3(N 1|N 2<N 2| A)$ would correspond to an ap boundary. The lowest boundary is perceived in $G 2$ and $G 4$ $(N 1|N 2<N 2| A)$, implying potentially a $p w$ boundary. Between $N 2$ and $A$, the strongest boundary perceived in $G 2$ and $G 3$ $(N 1|N 2<N 2| A)$ could reflect an ip boundary, and the weaker one perceived in $G 4(N 1|N 2<N 2| \boldsymbol{A})$ could correspond to an $a p$ boundary. The lowest boundary perceived in $G 1(N 1 \mid N 2>$ $N 2 \mid \boldsymbol{A})$ could also reflect a $p w$ boundary. These results are particularly interesting when considering the alleged propositions for French prosodic constituency. In most theoretical descriptions, indeed, French accent is said to be post-lexical, which makes it impossible to recognize a boundary level lower than the $a p$, namely the $p w$ level close to the lexical word $[5 ; 6 ; 17]$. Our results however indicate that such a constituency level is actually perceived: between $N 2 \mid A$ in $G 1$ and between $N 1 \mid N 2$ in $G 2$ and $G 4$.

Our investigations on Prominence also confirm these assumptions. Two main results emerge: first, $F A$ was clearly used to mark structures perceived as $G l$ at the ip boundary, which can be interpreted as an independent and "supporting role" [30] to the strong boundary scores found at this site. This result questions the proposition in [3] according to which boundary tones $(H \%)$ supersede FA pitch accent $\left(H^{*}\right)$ at higher prosodic boundaries. Namely, the syncretic occurrence of $F A$ prominence and intonation boundaries does not block the independent perception of both these prosodic events. The second important result concerns IA. The perception of IA does not depend on Grouping choices. More surprisingly with regards to the literature in French, it is not linked to constituents' length, contradicting the hypothesis that IA essentially plays a rhythmic role in French (see among others $[19 ; 21 ; 22])$. Rather, our results indicate that $I A$ is perceived as stronger than $F A$, with consistent high scores throughout the prosodic structure. Previous acoustic results suggested that $I A$ preferentially marks the lowest $a p$ level over $F A$ [23]. The present perceptual results rather point towards preferential marking of the $p w$. Indeed, results show that $I A$ after $p w$ boundaries in $G 1(N 2 \mid A)$, and in $G 2$ and $G 4(N 1 \mid N 2)$ was perceived as more salient than $F A$ (situated before the $p w$ boundary): here, $I A$ is a left boundary marker of constituency, starting as early as the $p w$ level.

We wish to elaborate on these results, which are particularly interesting to address the question of prosodic phrasing in French. New analyses are currently under way on a much larger database, using the same corpus (now 4 speakers) and the same tasks run on 80 listeners.

\section{Acknowledgements}

This study is supported by the Agence Nationale de la Recherche grant ANR-12-BSH2-0001 (PI: Corine Astésano) and by the Spanish Ministry of Economy and Competitiveness grant FFI2013-40419-P (PI: Lorraine Baqué). The authors also wish to thank Rafèu Sichel-Bazin for useful comments on an earlier version of this text. 


\section{References}

[1] M. Rossi, "Is Syntactic Structure Prosodically Retrievable," in Proceedings of 5th European Conference of Speech Communication and Technology, Rhodes, Greece, 1997, vol. 1 , pp. 1-8.

[2] M. Rossi, L'intonation, le système du français : description et modélisation. Paris, France: Editions OPHRYS, 1999.

[3] S.-A. Jun and C. Fougeron, "Realizations of accentual phrase in French intonation," Probus, vol. 14, no. 1, pp. 147-172, 2002

[4] E. Selkirk, "On derived domains in sentence phonology," Phonology, vol. 3, pp. 371-405, May 1986.

[5] M. Nespor and I. Vogel, Prosodic phonology, Foris. Dordrecht, 1986.

[6] B. Hayes, "The prosodic hierarchy in meter," in Rhythm and Meter, Academic Press., P. Kiparsky and G. Youmans, Eds. Orlando, Florida, 1989, pp. 201-260.

[7] B. Post, "Tonal and phrasal structures in French intonation," Thesis, The Hague, 2000.

[8] D. R. Ladd and N. Campbell, "Theories of Prosodic Structure: Evidence from Syllable Duration," in Proceedings of the 12th Congress of Phonetic Sciences, 1991, vol. 2, pp. 290-293.

[9] D. R. Ladd, Intonational Phonology. Cambridge, U.K: Cambridge University Press, 1996.

[10] E. Selkirk, "Sentence prosody: intonation, stress and phrasing," in The Handbook of Phonological Theory, Blackwell., J. Goldsmith, Ed. London, UK, 1995, pp. 550-569.

[11] R. Kager, Optimality theory. Cambridge, U.K: Cambridge University Press, 1999.

[12] E. Selkirk and K. Tateishi, "Constraints on Minor Phrase formation in Japanese," in Proceedings of the 24th Annual Meeting of the Chicago Linguistic Society, 1988, pp. 316-336.

[13] M. E. Beckman and J. B. Pierrehumbert, "Intonational structure in Japanese and English," Phonology, vol. 3, no. 01, pp. 255 309, May 1986

[14] S.-A. Jun and C. Fougeron, "A Phonological Model of French Intonation," in Intonation: Analysis, Modeling and Technology, Kluwer Academic Publishers, vol. 15, A. Botinis, Ed Dordrecht: Springer Netherlands, 2000, pp. 209-242.

[15] A. Di Cristo and D. Hirst, "Rythme syllabique, rythme mélodique et représentation hiérarchique de la prosodie du français," Trav. Interdiscip. Lab. Parole Lang. Aix-En-Provence TIPA, no. 15 , pp. 9-24, 1996 .

[16] A. Michelas and M. D'Imperio, "Durational cues and prosodic phrasing in French: evidence for the intermediate phrase," in Speech Prosody 2010 100881:1-4, Chicago, Illinois, 2010.

[17] E. Selkirk, "The prosodic structure of function words," in Signal to syntax: Prosodic bootstrapping from speech to grammar in early acquisition, John J. Mc Carthy, Mahwah, NJ: Blackwell Publishing Ltd, 1996, pp. 187-213.

[18] M. Rossi, "Le français, langue sans accent?," in L'accent en français contemporain, vol. 15, I. Fonagy and P. Léon, Eds. 1980, pp. 13-51.

[19] J. Vaissière, "Rhythm, accentuation and final lengthening in French," in Music, Language, Speech and Brain, Macmillan Press, J. Sundberg, L. Nord, and R. Carlson, Eds. London, UK, 1991, pp. $108-120$.

[20] M. E. Beckman, "Evidence for Speech Rhythms across Languages," in Speech Perception, Production and Linguistic Structure, Y. Tohkura, E. vatikiotis-Bateson, and Y. Sagisaka, Eds. Tokyo, Japan, 1992, pp. 457-463.

[21] V. Lucci, "Prosodie, phonologie et variation en français contemporain," Lang. Fr., vol. 60, no. 1, pp. 73-84, 1983.

[22] M. Rossi, "L'intonation et l'organisation de l'énoncé," Phonetica, vol. 42, no. 2-3, pp. 135-153, 1985.

[23] C. Astésano, E. G. Bard, and A. Turk, "Structural influences on initial accent placement in French," Lang. Speech, vol. 50, no. Pt 3, pp. 423-446, 2007.

[24] C. Astésano, R. Bertrand, R. Espesser, and N. Nguyen, "Dissociation between prominence and boundary phenomena in French: a perception study," in Proceedings of $p S$-prominenceS conference, Viterbo, Italie, 2013.
[25] A. Di Cristo, "La prosodie au carrefour de la phonétique, de la phonologie et de l'articulation formes-fonctions," Trav. Interdiscip. Lab. Parole Lang. Aix-En-Provence TIPA, vol. 23, pp. 67-211, 2004.

[26] J. Cole, Y. Mo, and S. Baek, "The role of syntactic structure in guiding prosody perception with ordinary listeners and everyday speech," Lang. Cogn. Process., vol. 25, no. 7-9, pp. 1141-1177, Sep. 2010.

[27] J. Cole, T. Mahrt, and J. I. Hualde, "Listening for sound, listening for meaning: Task effects on prosodic transcription," in Speech Prosody 2014, Dublin, Ireland, 2014, pp. 858-863.

[28] E. Delais-Roussarie and I. Feldhausen, "Variation in Prosodic Boundary Strength: a study on dislocated XPs in French," in Speech Prosody 2014, Dublin, Ireland, 2014, pp. 1052-1056.

[29] I. Lehiste, "Phonetic Disambiguation of Syntactic Ambiguity," J. Acoust. Soc. Am., vol. 53, no. 1, p. 380, 1973.

[30] P. J. Price, M. Ostendorf, S. Shattuck-Hufnagel, and C. Fong, "The use of prosody in syntactic disambiguation," J. Acoust. Soc. Am., vol. 90, no. 6, pp. 2956-2970, Dec. 1991. 